\title{
NON STANDARD SIZE IMAGE COMPRESSION WITH REVERSIBLE EMBEDDED WAVELETS
}

\author{
Abdul Sattar \\ Assistant professor, Electronics \& Telecommunication Engineering Department, B. N. College Of Engineering PUSAD \\ shaikhsattar@rediffmail.com
}

\begin{abstract}
The rapid growth of digital imaging applications, including desktop publishing, multimedia, teleconferencing and high definition television, (HDTV) has increased the need of effective image processing. While processing any data, it requires large memory space for storage, which ultimately increases the transmission time. In order to save memory space and speed up the rate of transmission of data over networks, data compression is essential. Technically all image data Compressed into two groups as lossless and lossy. Some information is lost in the lossy compression, especially for radiological images. In this paper Non standard size still images are compressed by CREW using MATLAB. It uses a new form of wavelet transform technology. It is pyramidal (similar to hierarchical) and progressive by nature. From results it is observed that CREW provides higher compression ratio as compared to JPEG compression with same quality of image. The PSNR is also in acceptable range.
\end{abstract}

The features make CREW an ideal choice for applications that require high quality and flexibility for multiple input and output environments, such as, medical imagery, fixed-rate and fixed-size applications.

Keywords-CREW, JPEG, Image Compression, JPEG2000

\section{INTRODUCTION}

Uncompressed multimedia data (graphics, audio and video) requires considerable storage capacity and transmission bandwidth. Despite of rapid progress in mass storage density, processor speeds, and digital communication system performance, demand for data storage capacity and datatransmission bandwidth continues to outstrip the capabilities of available technologies. The recent growth of data intensive multimedia based applications have not only sustained the need for more efficient ways to encode signals and images but have made compression of such signals central to storage and communication technology [1].

For still image compression, the JPEG [2] standard has been established. The JPEG compression encoding is based on Discrete Cosine Transform [3] scheme. First the image is divided into $8 \times 8$ block size and then DCT is applied. Due to this block based DCT scheme, the performance of these encoders generally degrades at low bit rates.

Over the past few years, a variety of powerful and sophisticated scheme for image compression with high compression ratio and good image quality have been developed and implemented. More recently, the wavelet transform has emerged as a cutting edge technology. Wavelet based coding [4] provides substantial improvements in picture quality at higher compression ratios. This paper introduces some basic concepts on image compression and the more popular wavelet based image-coding schemes. Further the performance of wavelet-based compression is compared with JPEG compression.

In this paper section (II) describes image compression techniques using CREW. In section (III) the algorithm implemented is described. In section (IV) experimental results of wavelet transform are given. The performance comparison is briefed in section (V) and section (VI) concludes the paper.

\section{COMPRESSION WITH REVERSIBLE EMBEDDED WAVELETS}

CREW is a completely new type of image compression system. CREW is the first lossless and lossy continuous-tone still image compression system. It is based on a lossless reversible wavelet transform and has embedded quantization before storing and transmitting the mage. To reconstruct the compressed image, it is decoded, dequantized and inverse transformed.

\section{A. Reversible Wavelets}

A reversible transform is an implementation of an exact reconstruction transform in integer arithmetic, such that a signal with integer coefficients can be losslessly recovered. An efficient reversible transform is one with transform matrix [5] of determinant $\approx 1[6]$.There are two reversible wavelet 
transforms are employed. The first one, the so-called Stransform is introduced in and used by and [6] among others. The second one, the TS-transform was introduced in [7].

Let $\mathrm{x}(0), \mathrm{x}(1), \mathrm{x}(2), \ldots$ be the input signal, and let $\mathrm{s}(0), \mathrm{s}(1), \ldots \ldots$ and $\mathrm{d}(0), \mathrm{d}(1), \ldots \ldots$ be the smooth and the detail outputs respectively. The smooth output and the detail output are the results of the applications of the low-pass and the highpass filters respectively.

The S-transform: The S-transform is defined by the outputs at a given index, $n$, is given in equ. (a).

$$
\left\{\begin{array}{l}
s(n)=\frac{L_{X(2 n)+x(2 n+1)}}{2} \\
d(n)=\frac{x(2 n)-x(2 n+1)}{2}
\end{array}\right.
$$

The factor of two in the transform coefficients addressing is the result of an implied subsampling by two. This transform is reversible and the inverse is given in equ. (b).

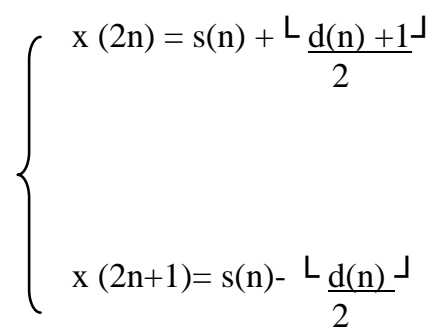

The TS-transform: Similar to the S-transform, the TStransform or two-six transform named after the number of taps in the low and high pass respectively

\section{B. Embedded Quantization}

Lossy compression is achieved by embedded quantization. Embedded is used here to indicate that the codestream includes the quantization. The actual quantization (or visual importance) levels can be a function of the decoder or the transmission channel, not necessarily the encoder.

\section{Pyramidal and Progressive Transmission}

The CREW wavelet is pyramidal. Here pyramidal refers to a decomposition by a factor of two of the image without difference images. Here progressive refers specifically to progressive by bit-plane, i.e. MSB followed by lessor bits. Both the spatial and wavelet domains can be decomposed progressively, although $\mathrm{CREW}$ is progressive in the wavelet domain specifically.

\section{Tree Structure of Wavelet Decomposition}

Figure.(1) shows a diagram of a wavelet decomposed image. The first level of coefficients is in the bottom and right most quadrants. These are denoted by LL, HL, HH, LH corresponding to high pass horizontal and low pass vertical, high pass horizontal and high pass vertical, low pass horizontal and high pass vertical respectively. The LL part of the first level is again decomposed, and so on. There is a natural and useful tree structure to wavelet coefficients in a pyramidal decomposition. Note that, there is a single LL subblock corresponding to the last level of decomposition. On the other hand there are as many HL, LH, and HH bands as the number of levels. The tree structure first introduced by Lewis and Knowles [8] and shown in figure (2), the coefficient at A is the direct parent to $\mathrm{B}, \mathrm{C}$ and $\mathrm{D}$ and all their decedents. Specifically B is the parent to the four coefficients around $\mathrm{E}$ and the sixteen coefficients around $\mathrm{H}$, etc

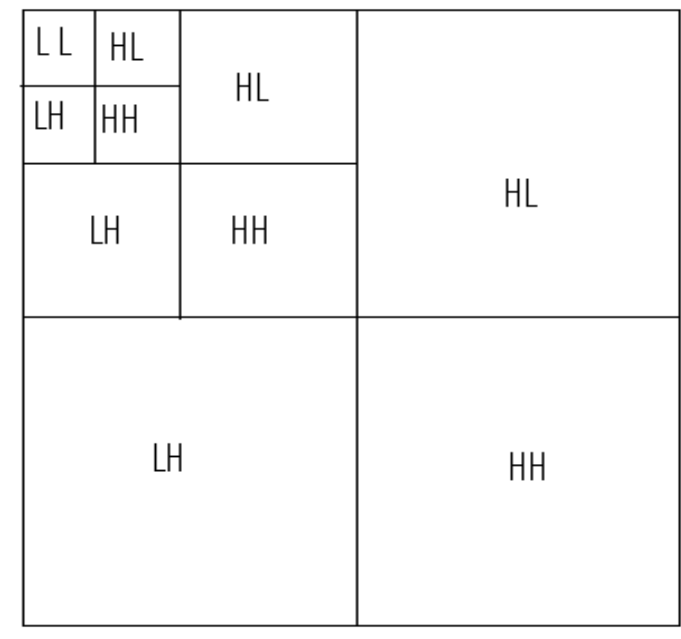

Figure.1Relationship between the coefficients

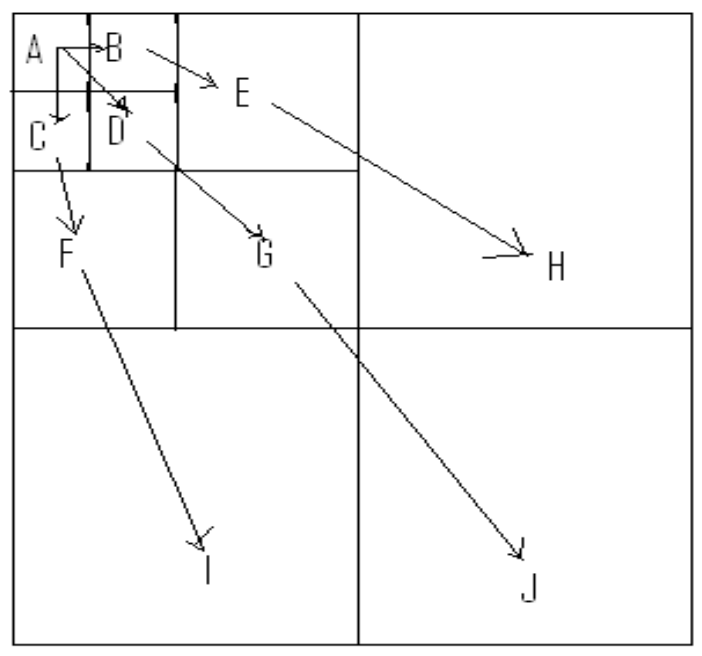

Figure.2 Tree Structure 


\section{E. Ordering Of The Coefficients And Bit Planes}

The ordering of the data is important to realize the embedded quantization of the codestream. Two ordering systems are required: one for ordering the coefficients and the second for ordering the binary values within a coefficient. This order leads to a bitstream that is coded with a binary entropy coder. All the coefficients within a coding unit are available in the random access memory, the embedding order between the coefficients within a coding unit can be any arbitrary order, known to both the encoder and the decoder. But since the entropy coder must be causal with respect to this ordering, it has a significant impact on the compression.

The embedded order used within a coefficient is bitsignificance in the transform domain which is similar to but more general than the system used in [9]. The largest possible coefficient is used as a reference, and the coefficients are aligned with respect to it. This alignment is near-optimal in terms of statistical error metric such as MSE. Other alignments could correspond to coefficient specific quantization.

The binary entropy coder uses a Horizon context model in order to encode the coefficients. The model uses bits within a coding unit based on the spatial and spectral dependencies of the coefficients. The available binary values of the neighboring coefficients and parent coefficients can be used to create contexts. The contexts however must be causal for decidability and in small numbers for efficient adaptation.

\section{F. Entropy Coding}

The bit stream is encoded with a binary entropy coder. For this implementation Q-coder is used.

\section{ALGORITHM}

1) Digitize the source image into a signal $s$, which is a string of numbers.

2) Decompose the signal into a sequence of wavelet coefficients w.

3) Use thresholding to modify the wavelet coefficients

4) Use quantization to convert w' to a sequence q.

5) Apply entropy coding to compress q into a sequence

\section{EXPERIMENTAL RESULTS}

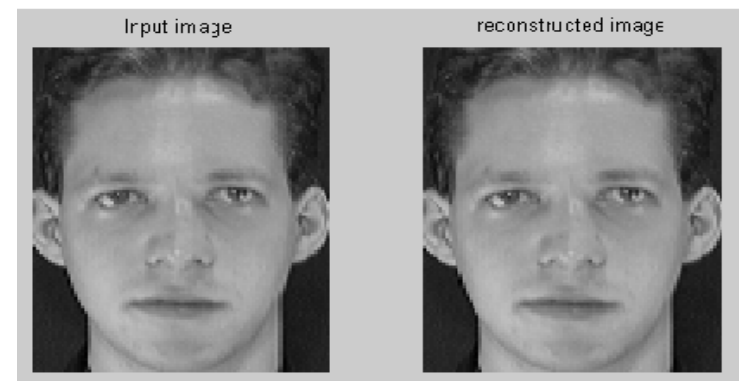

\section{PERFORMANCE COMPARISON CREW}

Table 1: Compression Ratios and PSNR using CREW

\begin{tabular}{|c|c|c|c|c|c|}
\hline Images & Level & MSE & $\begin{array}{l}\text { PSNR } \\
\mathrm{dB}\end{array}$ & C R\% & En-tropy \\
\hline face 1 & 3 & 7.95 & 39.13 & 87.50 & 7.03 \\
\hline $\operatorname{dog}$ & 3 & 0.89 . & 48.64 & 75 & 7.17 \\
\hline flower & 3 & 1.27 & 37.25 & 87.5 & 6.76 \\
\hline egret & 3 & 0.13 & 57.07 & 75 & 6.92 \\
\hline bird & 3 & 1.48 & 46.44 & 75 & 7.74 \\
\hline
\end{tabular}

From the images of the experimental results it is seen that in JPEG method the effects of artifacts are due the correlation between boundaries of the blocks of the DCT

\section{CONCLUSIONS}

Over the past several years, the wavelet transform has gained widespread acceptance in signal processing in general, and in image compression research in particular. In many applications wavelet-based schemes (also referred as subband coding) outperform other coding schemes like the one based on DCT. Since there is no need to block the input image and its basis functions have variable length, wavelet coding schemes at higher compression avoid blocking artifacts. Wavelet-based coding is more robust under transmission and decoding errors, and also facilitates progressive transmission of images. In addition, they are better matched to the HVS characteristics. Because of their inherent multiresolution nature[4], wavelet coding schemes are especially suitable for applications where scalability and tolerable degradation are important.

The CREW based image compression techniques provide higher compression ratios with moderate PSNR. The picture quality is also good for the intended application. The upcoming JPEG- 2000 standard incorporates the inherent multiresolution nature of wavelet transform. Many methods like EZW [6], SPIHT [7], EBCOT [8], SR [9] utilizes the wavelet image coding using decomposition techniques. There are few outstanding challenges facing the data compression community like interaction of harmonic analysis with data compression, joint source channel coding, image coding based on models of human perception, scalability, robustness, error resilience, and complexity. The challenges after fully resolved may enhance image data compression performance in the years to come. 


\section{REFERENCES}

[1] Subhasis Saha, "Image compression - from DCT to Wavelets: A review", ACM crossroads student magazine.

[2] Pennebaker, W. B. and Mitchell, J. L. JPEG - Still Image Data Compression Standards, Van Nostrand Reinhold, 1993

[3] Rao, K. R. and Yip, P. Discrete Cosine Transforms Algorithms, Advantages, Applications, Academic Press, 1990.

[4] Vetterli, M. and Kovacevic, J. Wavelets and Subband Coding, Englewood Cliffs, NJ, Prentice Hall, 1995.

[5] J. Shapiro, "An embedded wavelet hierarchical image coder," Proc. IEEE Int. Conf. Acoust., Speech, Signal Proc., vol. IV, pp. 657-660, March 1992.

[6] M. J. Gormish and J. Allen, "Finite state machine binary entropy coding," in Proc. Data Compression Conference, (Snowbird, Utah), p. 449, March 1993. Abstract only, full text available as Ricoh California Research Center Technical

[ 7 ] J. Villasenor, B. Belzer, and J. Liao, "Filter evaluation and selection in wavelet image compression," in Data Compression Conference, (Snowbird, Utah), IEEE, 1994.

[ 8 ] E. Simoncelli and E. Adelson, "Subband Transforms," in Subband Coding (J. Woods, ed.), ch. 4, Norwell, Massachusetts: Kluwer Academic Publishers, 1990.

[ 9 ] A. Zandi, J. D. Allen, E. L. Schwartz, and M. Boliek, "CREW: Compression with reversible embedded wavelets," in Data Compression Conference, (Snowbird, Utah), pp. 212-221, IEEE, March 1995. 